\title{
Factores de riesgo asociados a retraso del crecimiento intrauterino
}

\section{Risk factors associated with fetal growth restriction}

\author{
DOI: $10.46932 / \mathrm{sfjdv2n5-012}$
}

Received in: Jun1st, 2021

Accepted in: Sep 30th, 2021

\section{Ricardo Salas Flores}

Higher academic background: Endocrinólogo Pediatra., Master en Ciencias Médicas., Profesor Investigador.

Current Institution: Coordinación de Educación en Salud e Investigación. Hospital General Regional No. 6, Madero, Tamaulipas, Instituto Mexicano del Seguro Social, México. Facultad de Medicina "Dr.

Alberto Romo Caballero" Universidad Autónoma de Tamaulipas, Tampico, Tamaulipas, México.

Full address: Blvd. Adolfo López Mateos S/N, Col. Las Conchitas, Madero Tamaulipas.

\section{Brian González Pérez}

Higher academic background: Médico Familiar., Doctor en Ciencias de la Salud., Master en Ciencias Médicas., Master en Innovación y Tecnología Educativa., Profesor Investigador.

Current Institution: Servicio de Atención Familiar. Unidad Médica Familiar 38, Tampico Tamaulipas, Instituto Mexicano del Seguro Social, México. Facultad de Medicina "Dr. Alberto Romo Caballero"

Universidad Autónoma de Tamaulipas, Tampico, Tamaulipas, México.

Full address: Calle Argentina y Canadá 107 Col. Infonavit C.P. 89310, Tampico, Tamaulipas.

Email: brian.gonzalez.perez@gmail.com

\section{Verónica Olvera Mendoza}

Higher academic background. Doctora en Desarrollo Educativo., Master en Finanzas., Profesor Investigador.

Current Institution: Facultad de Medicina "Dr. Alberto Romo Caballero" Universidad Autónoma de Tamaulipas, Tampico, Tamaulipas, México.

Full address: Centro Universitario Sur, Cto. Interior S/N CP 89337, Tampico, Tamaulipas

\section{Raúl de León Escobedo}

Higher academic background: Médico Patólogo., Doctor en Ciencias de la Salud., Master en Investigación Clínica., Profesor Investigador.

Current Institution: Facultad de Medicina "Dr. Alberto Romo Caballero" Universidad Autónoma de Tamaulipas, Tampico, Tamaulipas, México.

Full address: Centro Universitario Sur, Cto. Interior S/N CP 89337, Tampico, Tamaulipas.

\section{Hebert Huerta Martínez}

Higher academic background: Endocrinólogo Pediatra.

Current Institution: Centro Médico Nacional Siglo XXI UMAE de Pediatría, México. Instituto Mexicano del Seguro Social, México.

Full address: Av. Cuauhtémoc 330 COl. Doctores, C.P. 06720 Alcaldía Cuauhtémoc de la Ciudad de México.

\section{Alejandra Salas Galarza}

Higher academic background: Médico Residente Endocrinologia. 
Current Institution: Centro Médico Nacional de Occidente, Guadalajara, Jalisco. Instituto Mexicano del Seguro Social, México.

Full address: Av. Belisario Domínguez 1000, Belisario Domínguez, CP 44329. Guadalajara, Jalisco.

\section{Wilberto Sánchez Márquez}

Higher academic background. Doctor en Desarrollo Educativo., Profesor Investigador. Current Institution: Facultad de Medicina "Dr. Alberto Romo Caballero" Universidad Autónoma de Tamaulipas, Tampico, Tamaulipas, México.

Full address (can be institutional or personal, as you prefer): Centro Universitario Sur, Cto. Interior S/N CP 89337, Tampico, Tamaulipas

\section{Jorge Pérez Ramírez}

Higher academic background. Master en Ciencias Médicas., Profesor Investigador. Current Institution: Facultad de Medicina "Dr. Alberto Romo Caballero" Universidad Autónoma de Tamaulipas, Tampico, Tamaulipas, México.

Full address (can be institutional or personal, as you prefer): Centro Universitario Sur, Cto. Interior S/N CP 89337, Tampico, Tamaulipas

\section{RESUMEN}

INTRODUCCION: El crecimiento de un feto se considera restringido si el peso es dos desviaciones estándar menor que el de la media correspondiente a su edad gestacional, está por debajo del percentil 3 para su misma edad gestacional, o se estanca, es decir, no alcanza el tamaño que le corresponde por su potencial biológico.

OBJETIVO: Identificar los factores de riesgo asociados con la restricción del crecimiento intrauterino en recién nacidos vivos.

METODOS: Estudio observacional, descriptivo, transversal y retrospectivo. Se revisaron los expedientes clínicos de madres cuyo producto haya nacido vivo con un peso igual o menor a 2500 gramos en el periodo comprendido de Enero del 2012 a Diciembre del 2013, en el Hospital General Regional N ${ }^{\circ} 6$ (HGR\#6) del IMSS. El análisis de los datos obtenidos se llevó a cabo mediante regresión lineal, estadística descriptiva y mediante medidas de tendencia central, para variables continuas, variables categóricas, porcentajes y frecuencias.

RESULTADOS: El análisis estadístico realizado demostró una asociación lineal significativamente fuerte de $94 \%$ entre las anormalidades placentarias y el RCIU ( $p=0.000)$ IC 95\%, DE 127.9-425.0. Así mismo existe asociación lineal moderada entre la Hipertensión arterial crónica y RCIU en un $47 \%(p=0.013)$ IC 95\% DE 25.9-205.5

CONCLUSIONES: Los factores de riesgo maternos son los que se asocian con mayor frecuencia a RCIU en recién nacidos vivos del HGR \#6 del IMSS.

Palabras clave: Crecimiento intrauterino, Parto prematuro, Restricción del crecimiento intrauterino, Peso al nacer, Desarrollo fetal.

\footnotetext{
ABSTRACT

INTRODUCTION: The growth of a fetus is considered restricted if the weight is two standard deviations less than that of the mean corresponding to its gestational age, is below the 3rd percentile for the same gestational age, or is stagnant, that is, it does not reach the size that corresponds to it due to its biological potential.

OBJECTIVE: To identify the risk factors associated with intrauterine growth restriction in live newborns. METHODS: Observational, descriptive, cross-sectional and retrospective study. The clinical records of mothers whose product was born alive with a weight equal to or less than 2500 grams in the period from January 2012 to December 2013 were reviewed at the Hospital General Regional $N^{\circ} 6$ (HGR \# 6) of the
} 
IMSS. The analysis of the data obtained was carried out using linear regression, descriptive statistics and measures of central tendency, for continuous variables, categorical variables, percentages and frequencies. RESULTS: The statistical analysis performed showed a strong significant linear association of $94 \%$ between placental abnormalities and intrauterine growth restriction (IUGR) $(p=0.000)$ 95\% CI, SD 127.9-425.0. Likewise, there is a moderate linear association between chronic arterial hypertension and IUGR in $47 \%$ ( $p=0.013) 95 \%$ CI SD 25.9-205.5

CONCLUSIONS: Maternal risk factors are those most frequently associated with IUGR in live newborns from HGR \# 6 of the IMSS.

Keywords: Intrauterine growth, Preterm birth, Intrauterine growth restriction, Birth Weight, Fetal Development.

\section{INTRODUCCION}

El retraso del crecimiento intrauterino es un término que se asigna a un recién nacido que no alcanzó su potencial de crecimiento, ya sea por factores genéticos o ambientales. El retraso en el crecimiento fetal es resultado del nacimiento de un neonato pequeño para la edad gestacional. La morbilidad y mortalidad ha incrementado en pacientes con peso pequeño para la edad gestacional comparados con aquellos con peso adecuado para la edad gestacional [1].

La definición más común de edad gestacional pequeña se refiere a una edad gestacional por debajo del percentil 10 [2]. Sin embargo, esta definición no hace distinción entre los pacientes pequeños por constitución anatómica, pequeños con retraso del crecimiento intrauterino y de tamaño adecuado con retraso de su potencial de crecimiento. Por ejemplo, al menos $70 \%$ de los fetos con peso por debajo del percentil 10 para la edad gestacional son pequeños por factores constitutivos como el sexo femenino, la raza materna, la paridad, o el índice de masa corporal; estos no proporcionan un riesgo elevado de morbilidad o mortalidad perinatal. La restricción moderada y severa del crecimiento fetal se definen como un peso al nacer entre el percentil 3 y el percentil 10, y un percentil menor a 3, respectivamente. Los recién nacidos de termino típicamente tienen un peso mayor a 2500gr hacia la semana 37 de gestación [2]. El índice ponderal es una proporción del peso corporal y la longitud expresada como:

$$
\mathrm{IP}=[\text { peso (en gr) x 100] } \div[\text { longitud }(\mathrm{en} \mathrm{cm})]
$$

Con el crecimiento normal, el índice ponderal aumenta gradualmente de la semana 30 a la 37 de gestación y después se mantienen constantes. El retraso en el crecimiento de tejido adiposo y de musculo esquelético, que contribuyen de manera importante para el peso corporal, resultan en un índice ponderal reducido. Las reducciones en el índice ponderal u otros índices como la proporción entre el perímetro braquial y la circunferencia occipito-frontal, pueden identificar retraso en el crecimiento en recién nacidos 
con peso por arriba de la percentil 10. El índice ponderal menor al percentil 10 refleja desnutrición fetal; el índice ponderal por debajo de la percentil 3 indica pérdida fetal severa [3].

Los datos antropométricos de los recién nacidos de diferentes edades gestacionales se han utilizado para generar curvas del crecimiento [3]. Estas curvas no son uniformes y pueden varias de 100 a 200 gr en cualquier edad gestacional. Los siguientes factores pueden influir en las variaciones:

- Diferencias en las características maternas, incluyendo edad, paridad, raza, grupo étnico, estado socioeconómico y habito corporal.

- Mediciones inadecuadas de la talla corporal y la estimación de la edad gestacional.

- Debido a que el nacimiento pretermino no es un acontecimiento normal, los factores patológicos que resultan en prematurez, pueden afectar el crecimiento fetal.

En general la realización de una óptima y personal curva de crecimiento en un feto específico, que cuenta con diferencias individuales (edad materna y grupo étnico) mejora la detección de un retraso del crecimiento intrauterino [4]. En un reporte, el uso de curvas del crecimiento en un estudio de Cohorte de 13,661 partos de feto único mejoró la habilidad de detectar retraso del crecimiento intrauterino y mejoró la habilidad para predecir algún resultado adverso [4]. Idealmente las curvas del crecimiento deberían de separarse y ser utilizadas en cada sexo porque los recién nacidos masculinos regularmente pesan más que las mujeres a la misma edad gestacional.

También, mediciones que manejen el peso al nacimiento de la madre y otros familiares pueden mejorar la clasificación de edad gestacional pequeña. Las curvas de crecimiento específico del grupo étnico también han sido desarrolladas, y su uso puede mejorar la identificación de neonatos con riesgo por morbilidad neonatal por retraso del crecimiento intrauterino [5].

Las curvas derivadas de medidas ultrasonográficas de fetos con edad gestacional conocida, quienes nacieron sanos y a término, proveen valores normativos para el crecimiento fetal [6]. En nacimientos pretérmino, la media estimada del peso fetal es mayor que la media derivada de recién nacidos vivos por edad gestacional porque los neonatos con peso bajo para la edad gestacional son más propensos a nacer prematuros que los de peso adecuado para la edad gestacional.

Como resultado, las curvas que estiman el peso fetal en comparación con las normas de peso al nacimiento, clasificará más neonatos con retraso del crecimiento intrauterino [7]

La incidencia del retraso del crecimiento intrauterino varía entre poblaciones e incrementa a menor edad gestacional. Aproximadamente el $10 \%$ de los neonatos a término en países desarrollados son pequeños para la edad gestacional, comparado con el $23 \%$ de los neonatos de termino en países en desarrollo. Los valores varían en neonatos con peso muy bajo (<1500gr) dependiendo si la recolección de los datos incluyen criterios para ambos; peso al nacer y edad gestacional. 
Los neonatos pequeños para la edad gestacional, tienen una restricción del crecimiento intrauterino simétrica o asimétrica. Los neonatos con RCIU simétrico tienen reducciones en el crecimiento del cuerpo y de la cabeza. El RCIU simétrico comienza en la edad gestacional temprana y normalmente es causada por factores intrínsecos, como infecciones congénitas o anormalidades cromosómicas. Sin embargo, el aporte nutricional disminuido tempranamente en el desarrollo puede restringir el crecimiento de todos los órganos.

Los neonatos con RCIU asimétrico tienen un cuerpo pequeño y una longitud y cabeza normal, el crecimiento anormal comienza típicamente en el segundo o tercer trimestre y resulta como una reducción en los nutrientes fetales, que limitan el glucógeno y el almacenamiento en grasa, sin embargo permite un crecimiento cerebral continuo. Mecanismos que separan el crecimiento cerebral son inciertos, pero pueden incluir el incremento del flujo sanguíneo cerebral.

La composición corporal está alterada en un neonato pequeño para la edad gestacional comparado con un apropiado para la edad gestacional. El total de grasa corporal, masa magra, y el contenido mineral del cuerpo se encuentran reducidos. El contenido de nitrógeno y proteínas es bajo debido a una disminución en la masa muscular. El contenido de glucógeno está disminuido en el músculo esquelético y en el hígado, por las bajas concentraciones plasmáticas de insulina y glucosa.

El retraso del crecimiento intrauterino puede ser causado por factores maternos, placentarios o fetales. Aproximadamente un tercio de los RCIU están asociados a causas genéticas, y los dos tercios restantes están relacionados al medio ambiente fetal. Sin embargo, una etiología subyacente no puede ser identificada en por lo menos $40 \%$ de los neonatos pequeños para la edad gestacional.

\section{Factores maternos}

-Inanición severa durante el embarazo

-Hipoxemia materna

-Enfermedades hematológicas e inmunológicas que causen trombosis de los espacios intervellosos y provoque una hipoperfusion uteroplacentaria.

-Enfermedad médica maternal (nefropatía, enfermedad vascular del colágeno) y complicaciones obstétricas (preeclampsia) asociadas con vasculopatía.

-Virus y parásitos (Rubeola, toxoplasmosis, citomegalovirus, varicela-zoster, malaria) que acceda al feto por vía transplacentaria o a través de las membranas fetales.

-Abuso de sustancias por parte de la madre, incluyendo el cigarro, alcohol y drogas ilícitas.

-Exposiciones tóxicas, incluyendo algunos medicamentos como warfarina, anticonvulsivos, agentes antineoplásicos y antagonistas del ácido fólico. 
-Grandes Alturas

-Variables demográficas incluyendo raza, embarazo en los extremos de la vida reproductiva, edad materna al nacimiento del primer hijo, nuliparidad o multiparidad y antecedentes previos de hijos con baja edad gestacional.

\section{Factores placentarios}

Cualquier desajuste entre la nutrición fetal o demanda respiratoria y el suministro placentario puede resultar en un crecimiento fetal alterado. El RCIU es resultado de una acumulación de lesiones fetales, como una vasculatura uteroplacental anormal, lesiones crónicas inflamatorias, desprendimiento prematuro de placenta, o anomalías estructurales de la placenta, como arteria umbilical única o hemangioma placentario.

\section{Factores neonatales}

-Anormalidades del cariotipo, como trisomías, alteraciones autosómicas, cromosomas anulares, etc. La presencia de anormalidades cromosómicas normalmente provocan un RCIU, más frecuentemente de tipo simétrico.

-Síndromes genéticos, como el síndrome de Bloom, enanismo, síndrome de Russell-Silver.

-Anomalías congénitas mayores: Por ejemplo enfermedades cardiacas congénitas, se ha estudiado que los recién nacidos con anormalidades estructurales del corazón es doblemente propenso a RCIU, en comparación con aquellos sin alteraciones estructurales.

-La relación entre el embarazo múltiple y el RCIU es directamente proporcional al número de fetos presentes. [8-11].

\section{Aspectos Clínicos}

Los neonatos con edad gestacional pequeña, son delgados, con piel descamada, masa muscular y tejido graso subcutáneo disminuido. El rostro tiene una típica apariencia arrugada y encogida, y el cordón umbilical regularmente es delgado. Puede existir presencia de meconio. En recién nacidos con RCIU asimétrico, la cabeza es relativamente más grande en comparación con el tamaño del cuerpo lo que causa dificultades en la transición cardiopulmonar, cursando con asfixia perinatal, aspiración de meconio o hipertensión pulmonar persistente, termorregulación alterada e hipoglucemia, policitemia y función autoinmune alterada. 
Manejo de paciente con RCIU

$\mathrm{Si}$ un feto es conocido con restricción del crecimiento, los problemas pueden anticiparse comenzando desde el nacimiento. Se debe investigar la etiología del RCIU, sin embargo en aproximadamente $40 \%$ de los casos no se puede identificar una causa. Se debe poner atención específicamente en malformaciones congénitas, anormalidades cromosómicas e infecciones. Debido a que los neonatos pequeños para la edad gestacional tienen una termorregulación alterada, se debe evitar la pérdida de calor mediante secado inmediato y colocación del neonato en una cuna radiante. La reanimación inmediata, incluyendo el limpiado de meconio de la vía aérea es necesaria. El tratamiento adecuado se inicia para alteraciones transitorias, incluyendo neumonía por aspiración de meconio, disfunción miocárdica, hipertensión pulmonar persistente.

La alimentación enteral debe iniciarse tempranamente en neonatos a volúmenes apropiados para el peso, incluyendo a los neonatos prematuros. En neonatos con asfixia perinatal severa, enfermos, o que no toleren la dieta enteral, la alimentación debe ser retenida y debe administrarse nutrición intravenosa. La monitorización de la hipoglucemia se inicia en la primera o segunda hora post parto. Las muestras son obtenidas antes de la alimentación. La vigilancia se continua en pacientes con bajas concentraciones de glucosa en el plasma $(<40 \mathrm{mg} / \mathrm{dL})$ hasta que se comience la alimentación enteral y se normalicen los niveles de glucosa. Los neonatos pequeños para la edad gestacional, que son prematuros o presentaron asfixia al nacimiento, tienen riesgo de Hipocalcemia. Se deben monitorizar los niveles de calcio sérico $>12 \mathrm{hr}$ post parto y se deben administrar dosis adecuadas de calcio. En comparación con neonatos con edad gestacional adecuada, los que desarrollaron restricción del crecimiento intrauterino son más propensos a presentar:

- Dificultad en la escuela o necesidad de educación especial.

- Disfunciones neurológicas

- Problemas del comportamiento (Déficit de atención con hiperactividad).

Falla del crecimiento $[9,10]$.

La identificación de los factores de riesgo predisponentes a retraso del crecimiento intrauterino nos permitirán conocer de manera temprana (previos al trabajo de parto) los posibles daños que presentará el producto, esto nos permite prevenir el daño al iniciar con tratamiento oportuno, disminuyendo la mortalidad y efectos a largo plazo y así mejorar la calidad de vida de estos pacientes en el futuro, disminuyendo los riesgos asociados con el RCIU y patologías cardiovasculares, alteraciones neurológicas y psicosociales. El objetivo de este estudio fue identificar los factores de riesgo asociados con la restricción del crecimiento intrauterino en recién nacidos vivos. 


\section{METODOS}

Estudio observacional, descriptivo, transversal y retrospectivo, realizado en el Hospital General Regional $N^{\circ} 6$ del Instituto Mexicano del Seguro Social en Cd. Madero, Tamaulipas. Se revisaron expedientes clínicos de pacientes de sexo femenino atendidas en sala de maternidad, de las cuales se obtuvo producto nacido vivo con peso menor o igual a 2500 gramos en el periodo de tiempo comprendido de enero del 2012 a diciembre del 2013. En una hoja de recolección de datos, se recabó la siguiente información: Nombre de la madre, número de afiliación, edad, escolaridad, tabaquismo, alcoholismo, enfermedades maternas como diabetes mellitus tipo 2, diabetes gestacional, hipertensión arterial sistémica, enfermedad tiroidea, unidad de medicina familiar, número de consultas prenatales, gesta, parto, cesárea, aborto, fecha de última regla, fecha última de parto, amenaza de parto pretérmino, retraso del crecimiento intrauterino previo, periodo intergenésico, consumo de ácido fólico, patologías durante el embarazo, fecha de nacimiento del producto, hora, sexo, semanas de gestación, talla, peso, Apgar, Silverman Anderson, puntuación por Capurro, anomalías de la placenta/cordón umbilical, anomalías congénitas. Se determinaron los nacidos vivos con peso bajo (menor o igual a 2500 gramos), así como el número de factores de riesgo para restricción del crecimiento intrauterino, posteriormente se clasificaron los pacientes con RCIU de acuerdo a las tablas Fenton de la CDC, 2006. El protocolo de investigación fue aprobado por el Comité Local de Investigación en Salud 2801 del Instituto Mexicano del Seguro Social. El análisis de los datos obtenidos se llevó a cabo mediante regresión lineal, estadística descriptiva y mediante medidas de tendencia central, para variables continuas, variables categóricas, porcentajes y frecuencias, se utilizó el programa estadístico SPSS (V.23; SPSS).

\section{RESULTADOS}

La mayor incidencia de casos de retraso en el crecimiento intrauterino se observó en madres mayores de 35 años, correspondiente a un $2.72 \%$ del total de nacidos vivos de ese grupo de edad, comparado con un 1.48\% en el grupo de 21-34 años, y un 1.27\% en madres jóvenes menores de 20 años, lo que nos dice que este grupo de madres (>35 años); tienen el doble de riesgo relativo de engendrar productos con retraso en el crecimiento intrauterino (Gráfica 1). 
Gráfica 1. Relación de RN vivos con PAEG / Casos de RCIU por edad materna

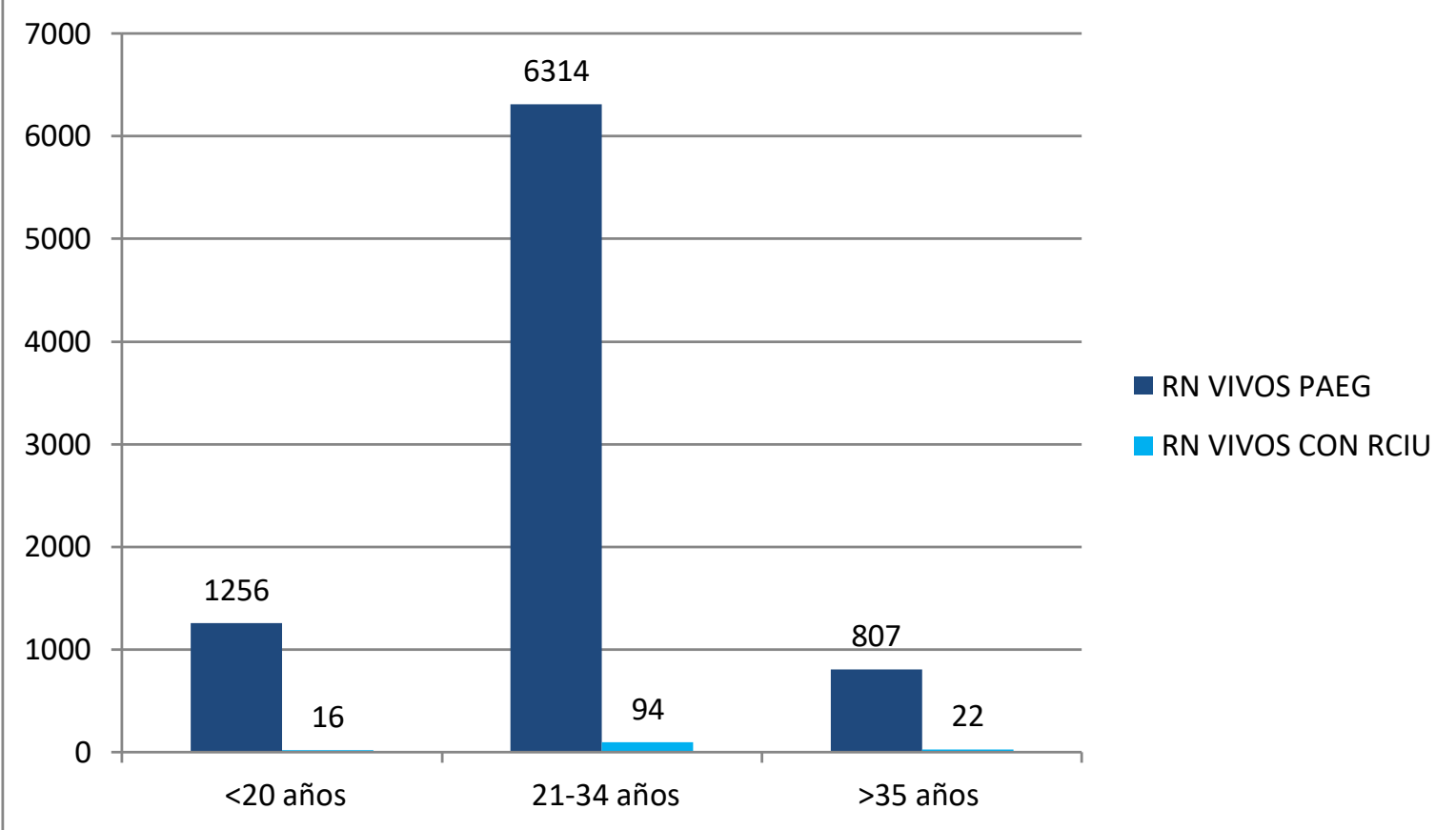

Nota: $\mathrm{RN}=$ Recién nacido, PAEG= Producto acorde a edad gestacional, RCIU= Retraso en el crecimiento intrauterino.

La media de peso al nacimiento también fue menor en productos de madres >35 años (1699 gramos); no siendo así en los otros dos grupo con 1979 gramos y 2016 gramos respectivamente, esto por la importante hipoperfusión tisular a nivel placentario adquirida con la edad, lo que se ve reflejado en el peso al nacimiento (Gráfica 2).

Gráfica 2. Peso de productos con RCIU por edad materna.

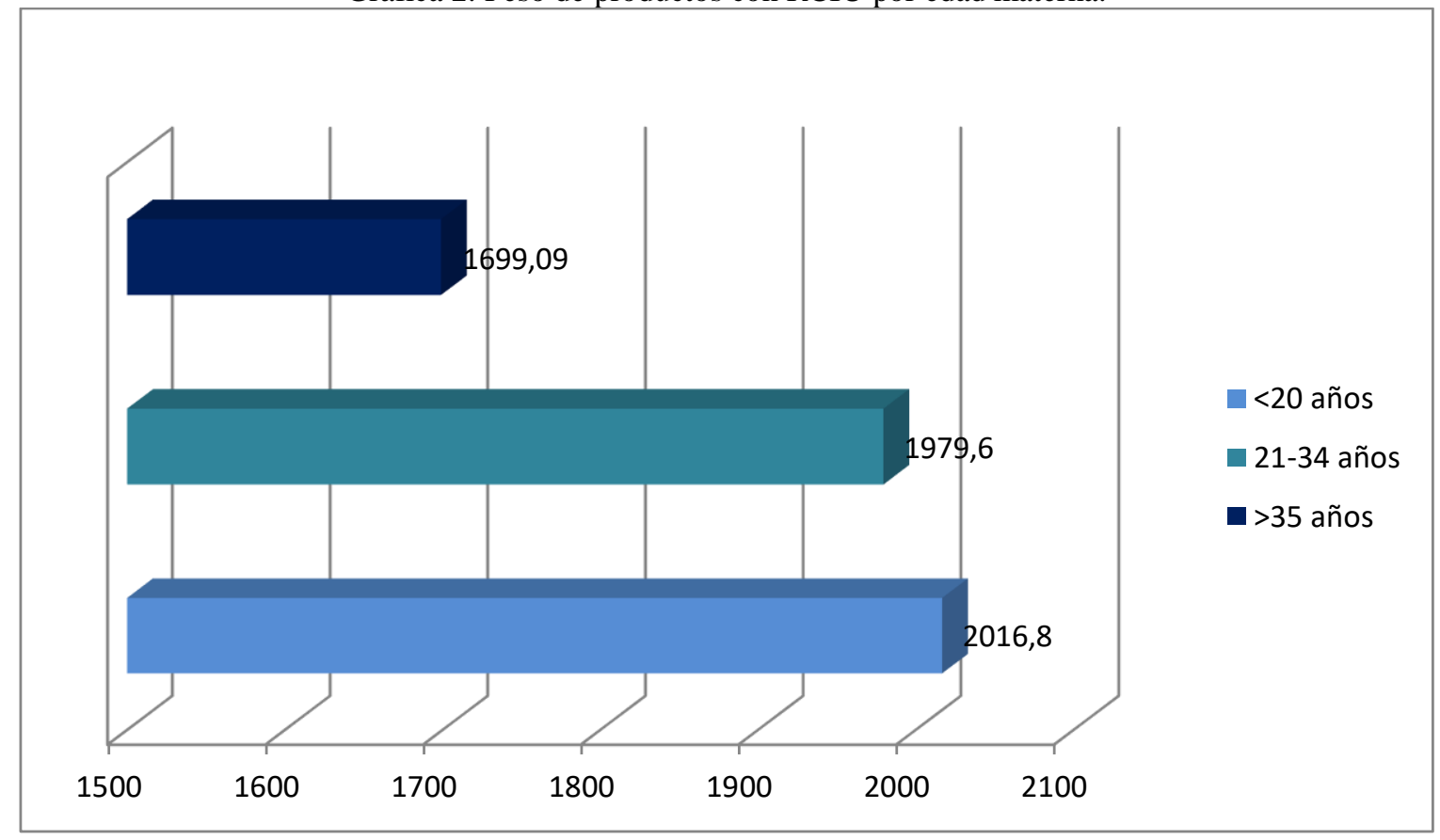


Así mismo la talla media de los productos al nacer, fue mucho más baja en productos hijos de madres > 35 años $(40.6 \mathrm{~cm})$, percatándonos con esto de un retraso en el crecimiento intrauterino simétrico que prevalece a esta edad materna (Gráfica 3).

Gráfica 3. Talla en productos con RCIU por edad materna.

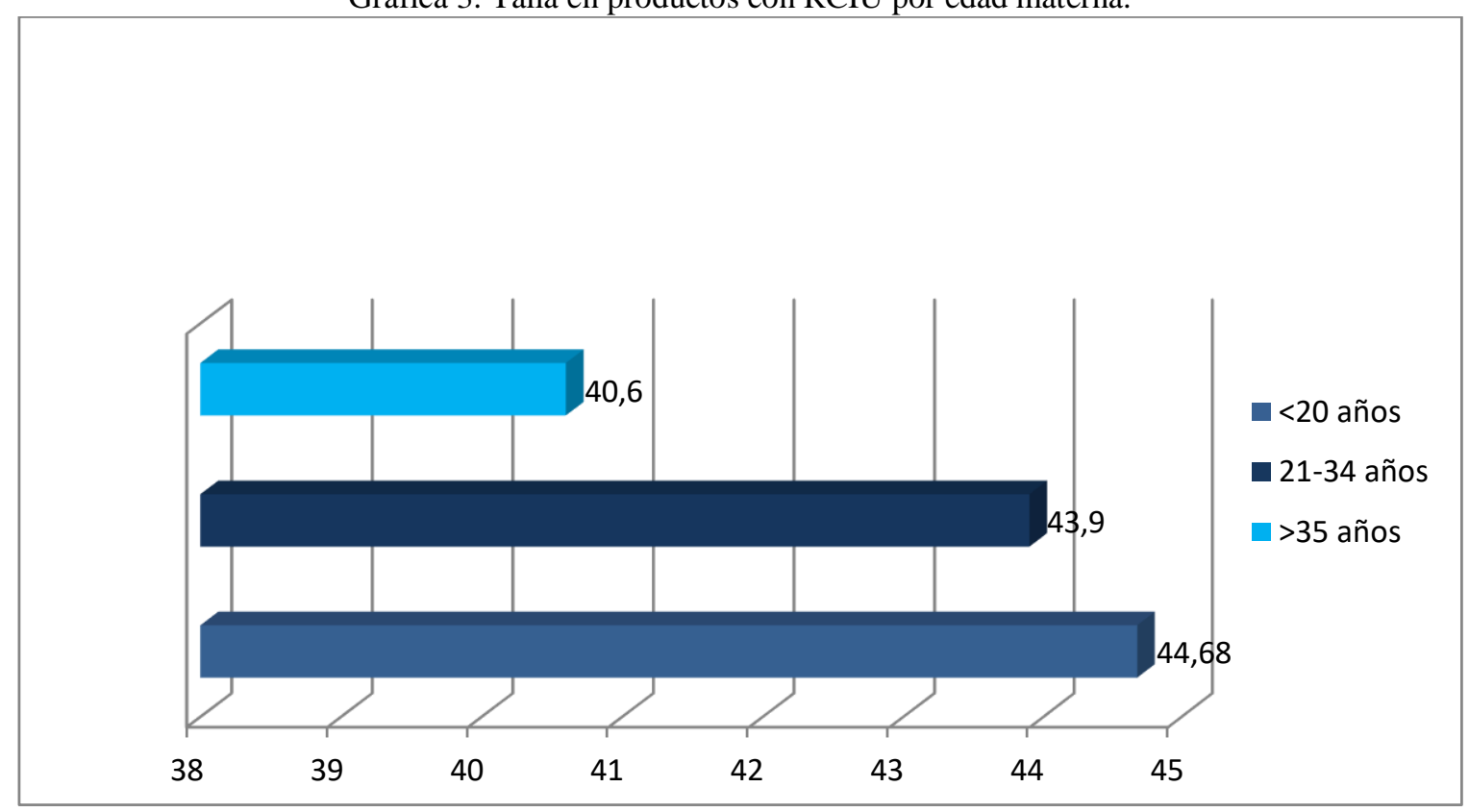

En los tres grupos de madres, predomino el RCIU simétrico observándose una alza notoria en el grupo de madres $>35$ años $(90.9 \% \%)$ esto debido a que los factores maternos asociados a RCIU en esta edad comienzan a presentarse desde el primer trimestre de gestación, dando como resultado un peso y talla por debajo del percentil 10. No así en el grupo de madres <20 años, donde se observó la mayor prevalencia de $<$ RCIU asimétrico con un $25 \%$, ya que los factores maternos más frecuentes a esta edad, empiezan a presentarse a partir del segundo trimestre del embarazo, dando como resultado final un peso bajo, pero talla normal (Gráfica 4). 
Gráfica 4. Casos de RCIU simétrico y asimétrico por edad materna.

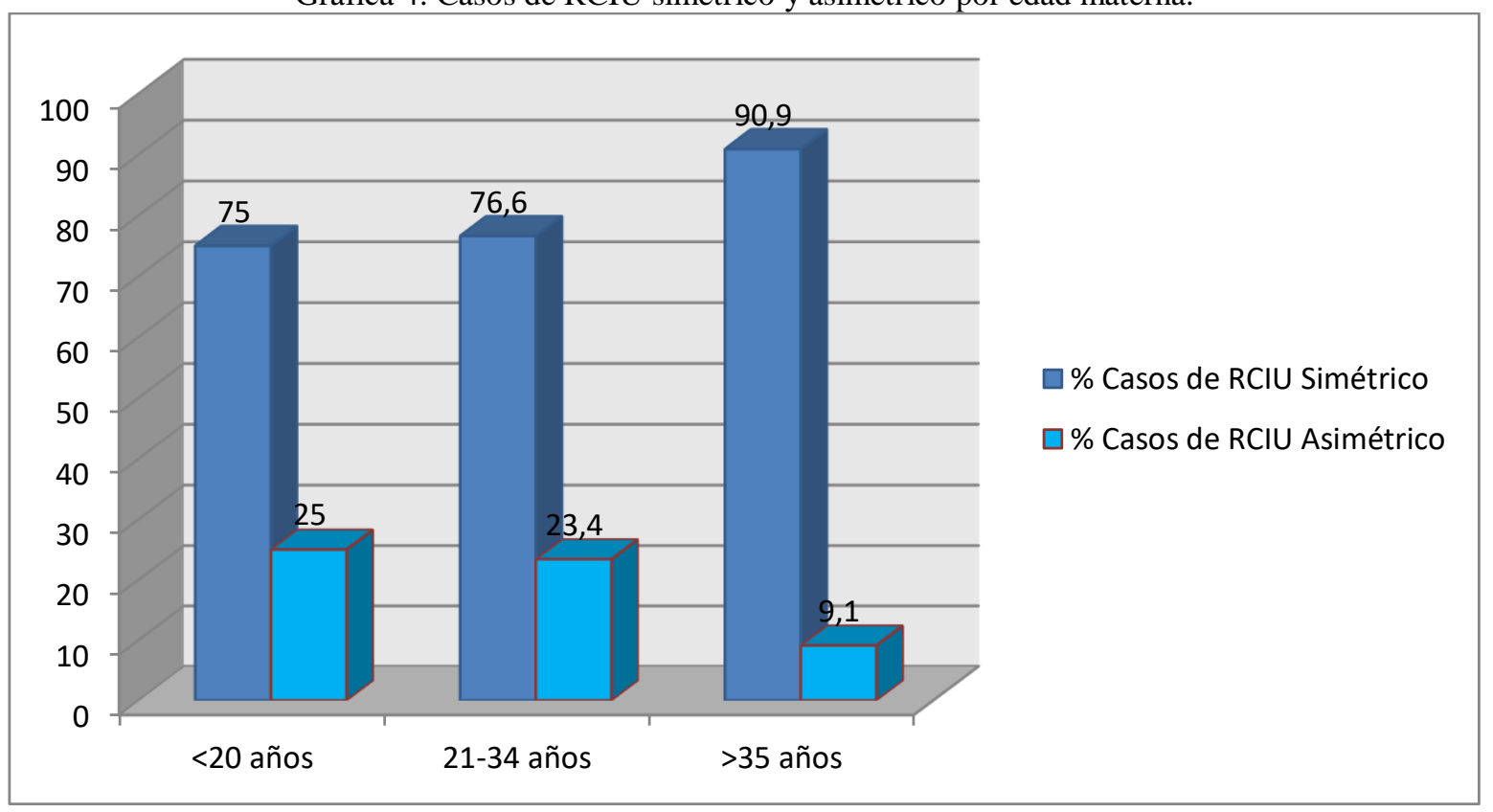

El retraso en el crecimiento intrauterino se presentó con más frecuencia en el sexo masculino en un $61 \%$, siendo de la siguiente manera por grupo de edad materna, <20 años (81.25\%), de 21-34 años $(57.5 \%)$ y en $>35$ años $(59.1 \%)$. La escolaridad materna no fue una variable de importancia para este estudio, ya que no hubo un resultado significativo en la misma. El control prenatal deficiente definido por la NOM-007-SSA2-1993 para la Atención de la mujer durante el embarazo, parto y puerperio y del recién nacido, como menos de 5 consultas, fue un factor importante en madres $>35$ años, donde dicho control deficiente se observó hasta en un 27.2\%. El periodo intergenésico, en madres de >35 años se observó que fue largo >4 años hasta en un 59.1\%, siendo solo significativo en este grupo ya que al aumentar el PI aumentaron su edad y por lo tanto el riesgo de RCIU. Al contrario, en mujeres <20 años se obtuvo que tuvieron la mayor incidencia de periodo intergenésico corto $<2$ años, lo que esto explicaría la presencia de RCIU por disminución del estado nutricional, dando un RCIU asimétrico como ya se demostró anteriormente (Gráfica 5). 
Gráfica 5. Periodos intergenésicos por grupos de madres con RCIU.

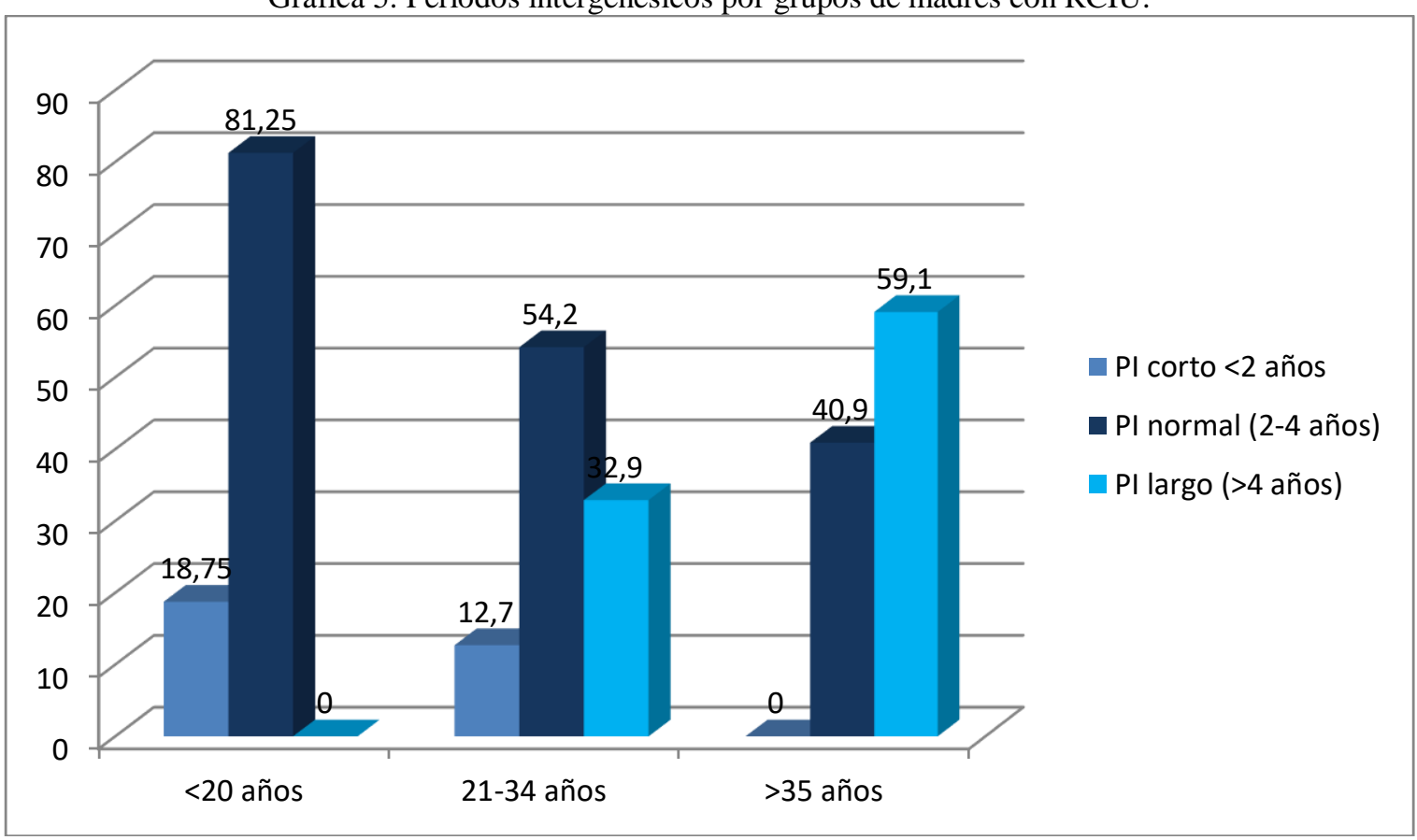

Nota: PI= Periodo intergenésico.

La historia de retraso en el crecimiento intrauterino en productos anteriores, prevaleció en madre de 21-34 años de edad en un 19.1\%, comparado con un $6.25 \%$ en <20 años y un $9.09 \%$ en madres mayores de 35 años de edad. Hubo relación de retraso en el crecimiento intrauterino y amenaza de parto prematuro en un $45.4 \%$ en madres de >35 años, $37.5 \%$ en menores de 20 años y un $20.2 \%$ en madres en edad ideal reproductiva. El alcoholismo solo se relacionó a RCIU en un 5.3\%, así mismo el alcoholismo en menor proporción solo un $3.1 \%$, no siendo las toxicomanías maternas un factor frecuente asociado a RCIU en nuestra población.

La hipertensión arterial sistémica crónica, se encontró hasta en un $13.6 \%$ de las madres $>35$ años, como enfermedad cronicodegenerativa propiamente; y en 9.5\% en madres de 21-34 años de edad, siendo este un factor asociado a tener con producto final un recién nacido con RCIU (Gráfica 6). 
Gráfica 6. Madres de productos con RCIU portadoras de hipertensión arterial crónica.

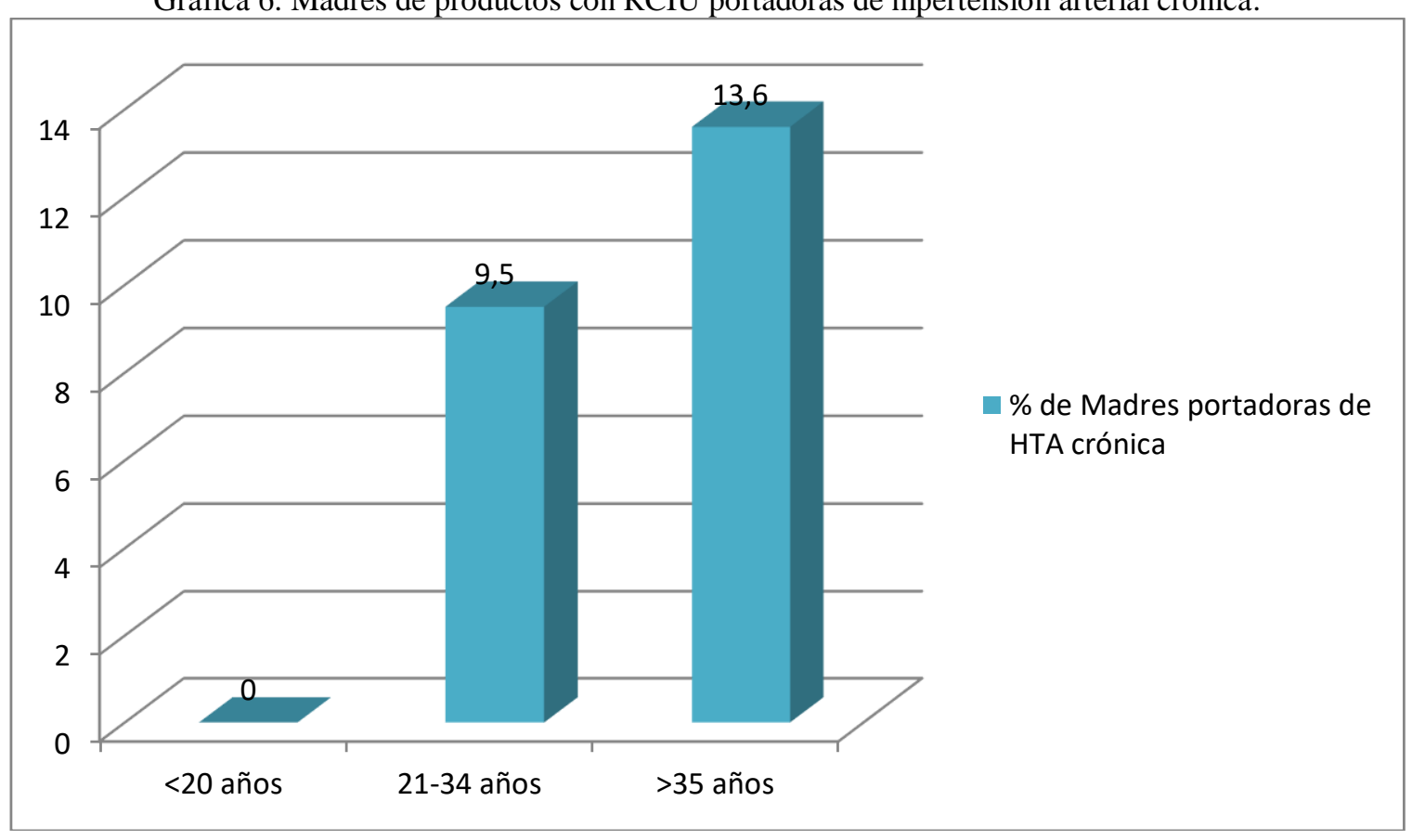

La toxemia materna se presentó en los tres grupos de edad con un 36.3\% (>35 años), 36.1\% (21 34 años) y $18.75 \%$ (<20 años), comprobando con esto la asociación existente en condicionar un peso bajo al nacer en productos cuyas madres sean portadoras en algún momento de su gestación de cualquiera de las variedad de toxemia materna (Enfermedad hipertensiva inducida por el embarazo, preeclampsia y eclampsia). (Gráfica 7).

Gráfica 7. Mujeres que tuvieron hipertensión inducida por el embarazo y productos con RCIU.

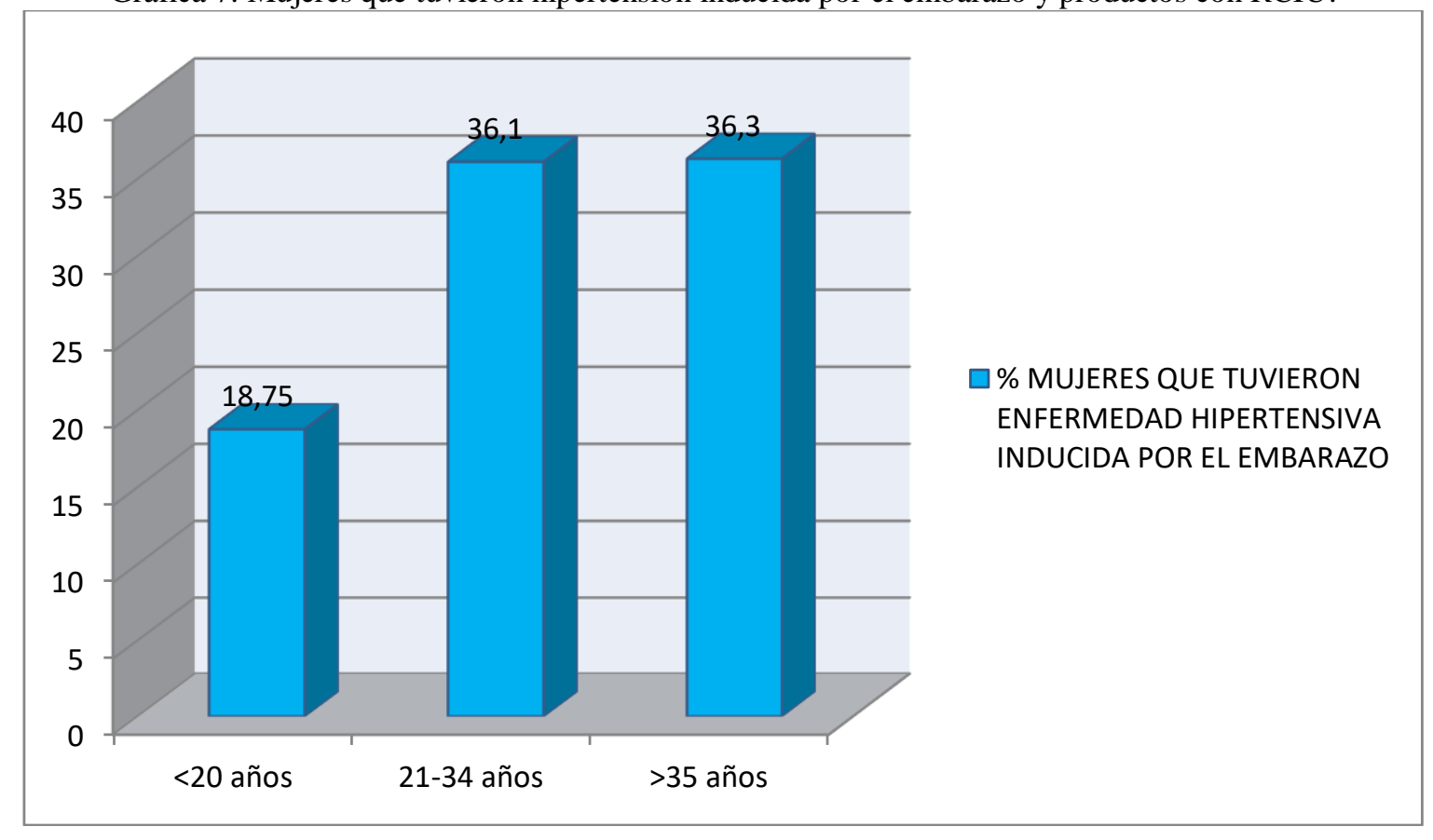


Patologías infecciosas materna como son las infecciones de vías urinarias y cervicovaginales, se presentaron de manera importante en los tres grupos de edad, siendo mayor las IVU con un $75 \%$ en el grupo de <20 años, y las cervicovaginales con un $43.75 \%$ en el mismo grupo (Gráfica 8).

Gráfica 8. Madres que presentaron patología infecciosa y productos con RCIU.

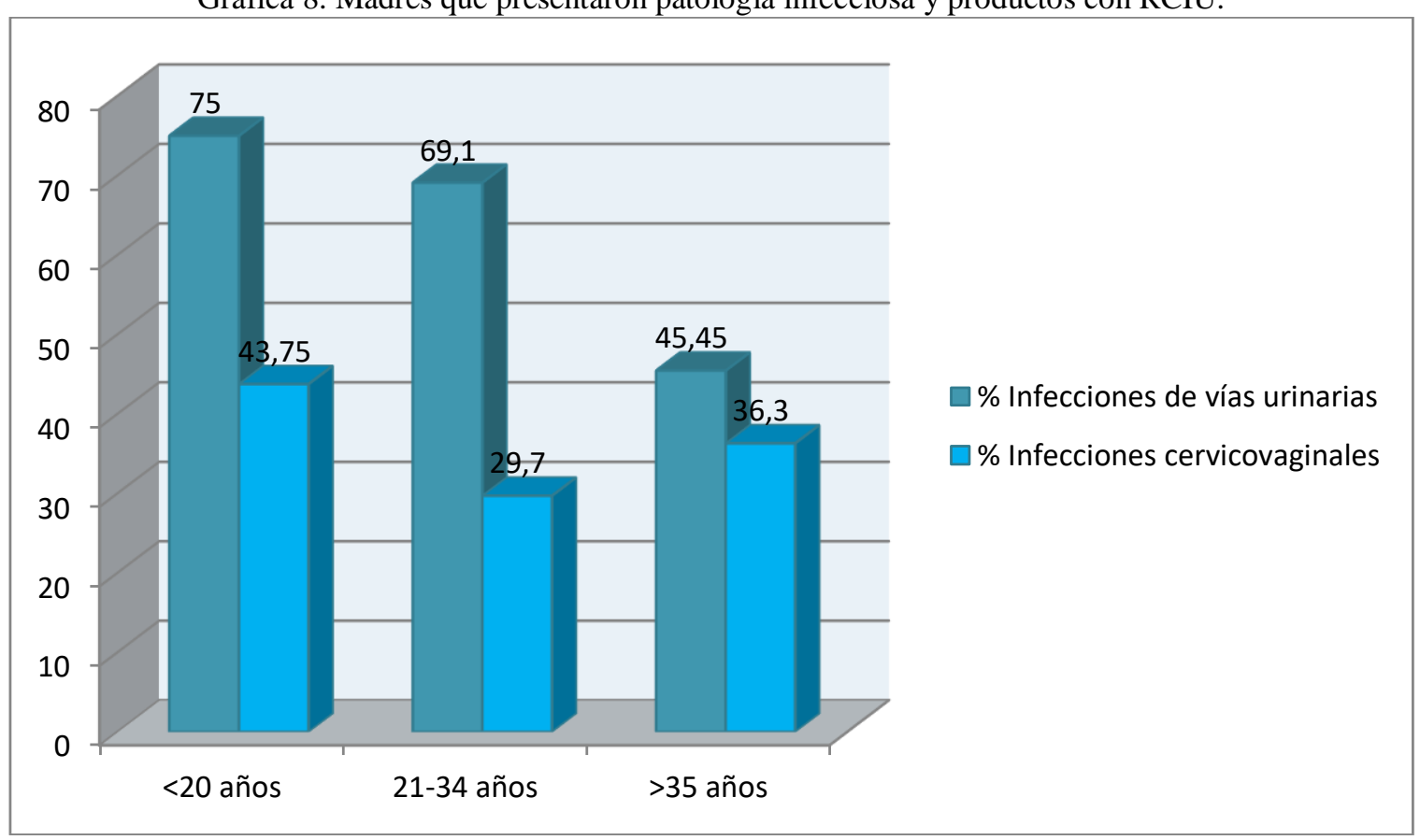

Las anormalidades placentarias se asociaron a RCIU en un $31.8 \%$ en madres añosas $>35$ años, esto por los cambios propios por la edad, así como la mayor indicencia de toxemia materna e Hipertensión arterial crónica, siendo la más frecuente de estas el desprendimiento prematuro de placenta normoinserta (Gráfica 9). Finalmente y no menos importante la anemia materna (Hemoglobina <12 gr/dL); se presentó con un mayor porcentaje en el grupo de madres de 21-34 años con un 39.3\%, seguida de un $31.25 \%$ de las madres $<20$ años y un $27.2 \%$ en madres $>35$ años. 
Gráfica 9. Madres con anormalidades de la placenta y RCIU.

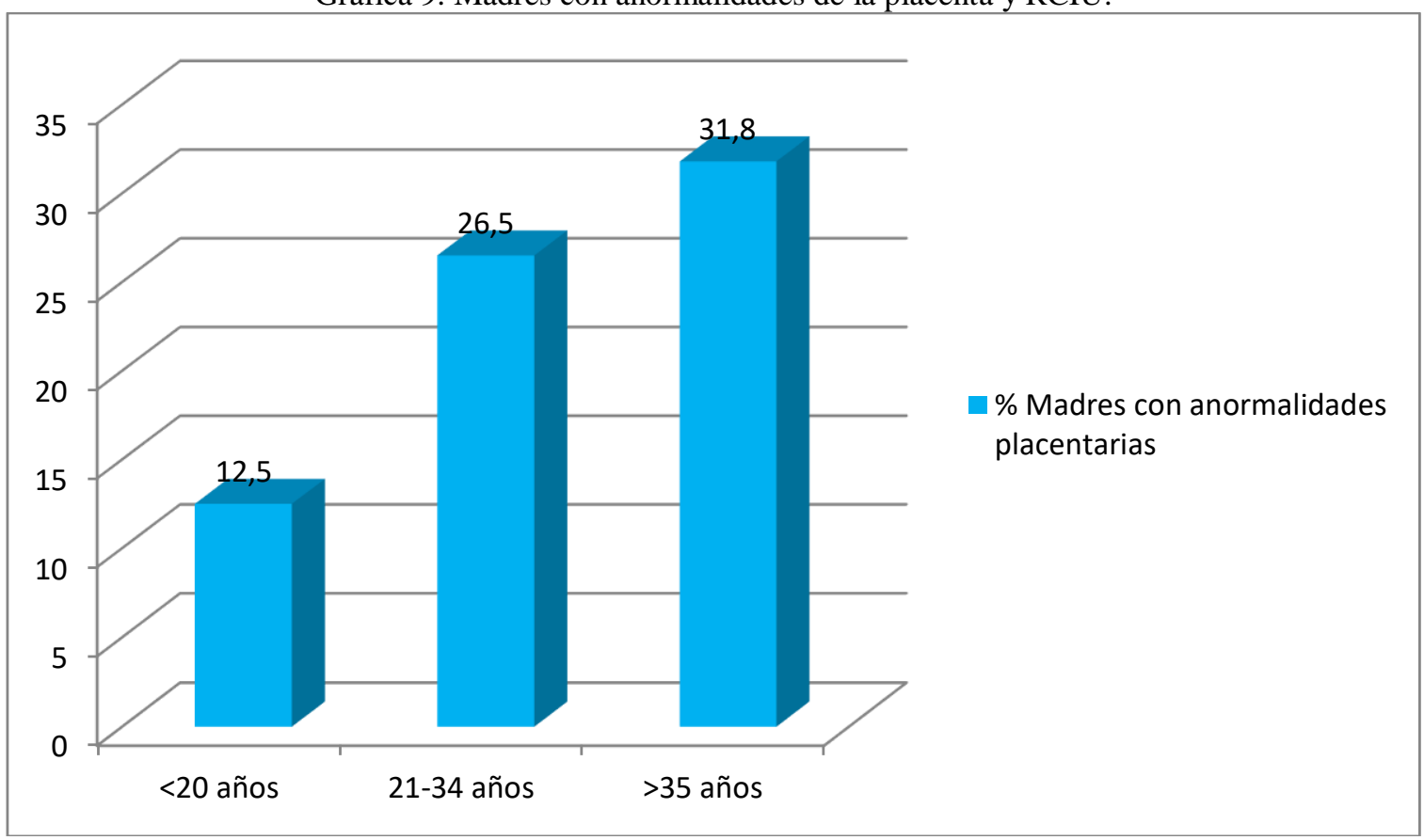

El análisis estadístico realizado demostró una asociación lineal significativamente fuerte de $94 \%$ entre las anormalidades placentarias y el retraso en el crecimiento intrauterino $(p=0.000)$ IC 95\%, DE 127.9-425.0. Así mismo existe asociación lineal moderada entre la Hipertensión arterial crónica y RCIU en un 47\% ( $p=0.013)$ IC 95\% DE 25.9-205.5.

\section{DISCUSIÓN}

En el presente estudio se identificó que una de las principales enfermedades de la edad adulta como es la hipertensión arterial sistémica crónica, tuvo una asociación lineal moderada con retraso en el crecimiento intrauterino en un 47\% ( $p=0.013)$ IC 95\% DE 25.9-205.5, esto debido a que las adaptaciones fetales invocados cuando el suministro de nutrientes maternoplacental no coincide con la demanda de nutrientes del feto, ocasionando RCIU como se menciona Grodfrey KM y cols.

La organización mundial de la Salud informa para México una prevalencia de retraso en el crecimiento intrauterino del $12 \%$. Nuestro estudio encontró $1.58 \%$, mucho menor a lo esperado, lo cual puede atribuirse a factores como la definición que se adopte respecto al diagnóstico de retraso del crecimiento intrauterino, distintas formas de elaboración de curvas de normalidad de peso y talla para recién nacidos y procedimientos utilizados para el cálculo de la edad gestacional. Kramer menciona que el sexo femenino del recién nacido es un factor de riesgo para retraso del crecimiento intrauterino, en nuestro estudio no mostró asociación estadística, siendo además más frecuente en el sexo masculino con un $62.8 \%$. 
En cuanto a la edad materna, el grupo más afectado en nuestra investigación fue el de mujeres mayores de 35 años con un $2.72 \%$ de casos de RCIU, lo que no correlaciona con el estudio publicado por Gomella, donde el grupo más afectado fue el de mujeres menor de 20 años. La escolaridad nula o de nivel básico no mostró asociación estadística como factor de riesgo para retraso en el crecimiento intrauterino, lo que no concuerda con investigaciones publicadas donde las pacientes con mala situación económica tienen habitualmente baja escolaridad, lo que condiciona desconocimiento de la físiología de la reproducción e inadecuado control médico durante el embarazo.

El grupo de control prenatal inadecuado (menos de 5 consultas) demostró un riesgo 4 veces mayor de retraso en el crecimiento intrauterino en relación con grupo que presentó control prenatal adecuado. Si a esto adicionamos escolaridad y estado socioeconómico bajos, es posible que durante el embarazo se pase por alto complicaciones que pudieran identificarse a tiempo.

De las complicaciones como la enfermedad hipertensiva del embarazo, infecciones de vías urinarias y cervicovaginales, así como la anemia materna, no demostraron en nuestro estudio significancia estadística asociadas como causa de retraso del crecimiento intrauterino, contrario a lo observado en el estudio de Arriola-Ortiz y cols. Nuestro estudio demostró una asociación lineal significativamente fuerte de $94 \%$ entre las anormalidades placentarias y el retraso en el crecimiento intrauterino.

\section{CONCLUSIONES.}

Los factores de riesgo maternos son los que se asocian con mayor frecuencia a retraso en el crecimiento intrauterino en recién nacidos vivos en el Instituto Mexicano del Seguro Social, Hospital General Regional No. 6 “Lic. Ignacio García Téllez”. Las anormalidades placentarias y la hipertensión arterial sistémica crónica son los factores maternos más frecuentemente asociados como causa de retraso en el crecimiento intrauterino. Es importante identificar al inicio del embarazo a las pacientes con algún factor de riesgo para desarrollar retraso del crecimiento intrauterino y llevar a cabo un buen control prenatal, con un manejo adecuado de las posibles complicaciones médicas del embarazo y así disminuir la morbilidad y mortalidad del recién nacido [21]. Podemos concluir señalando que es innegable la influencia de los factores maternos como causa de RCIU, por lo que debe constituir la diana de los esfuerzos que el médico y la enfermera deben desarrollar en su prevención, sobre todo en la comunidad. 


\section{REFERENCIAS BIBLIOGRÁFICAS}

1. L. Cabrero-D.Saldivar-E.Cabrillo, Obstetricia y medicina materno fetal, Edición 2007, Editorial: Médica Panamericana S.A. Capítulo:113, Paginas 939-945.

2. Mandy GT, MD. Small for gestional age infants. Uptodate, 2013, November.

3. Divon MY, MD. Fetal Growth Restriction: Diagnosis. Uptodate. 2013, August.

4. Figueras F, Figueras J, Meler E, et al. Customised birthweight standards accurately predict perinatal morbidity. Arch Dis Child Fetal Neonatal Ed 2007; 92:F277.

5. Skjaerven R, Gjessing HK, Bakketeig LS. New standards for birth weight by gestational age using family data. Am J Obstet Gynecol 2000; 183:689.

6. Di Battista E, Bertino E, Benso L, et al. Longitudinal distance standards of fetal growth. Intrauterine and Infant Longitudinal Growth Study: IILGS. Acta Obstet Gynecol Scand 2000; 79:165.

7. Cooke RW. Conventional birth weight standards obscuree fetal growth restriction in preterm infants. Arch Dis Child Fetal Neonatal Ed 2007; 92:F189.

8. Malik S, Cleves MA, Zhao W, et al. Association between congenital heart defects and small for gestational age. Pediatrics 2007; $119: \mathrm{e} 976$.

9. Guellec I, Lapillonne A, Renolleau S, et al. Neurologic outcomes at school age in very preterm infants born with severe or mild growth restriction. Pediatrics 2011; 127:e883.

10. De Jesus LC, Pappas A, Shankaran S, et al. Outcomes of small for gestational age infants born at $<27$ weeks' gestation. J Pediatr 2013; 163:55.

11. Divon MY, MD. Fetal Growth Restriction: Causes and risk factors. Uptodate. 2014, January

12. Malloy MH. Size for gestational age at birth: impact on risk for sudden infant death and other causes of death, USA 2002. Arch Dis Child Fetal Neonatal Ed 2007; 92:F473.

13. Doctor BA, O'Riordan MA, Kirchner HL, et al. Perinatal correlates and neonatal outcomes of small for gestational age infants born at term gestation. Am J Obstet Gynecol 2001; 185:652.

14. Peacock JL, Lo JW, D'Costa W, et al. Respiratory morbidity at follow-up of small-for-gestationalage infants born very prematurely. Pediatr Res 2013; 73:457.

15. Zeitlin J, El Ayoubi M, Jarreau PH, et al. Impact of fetal growth restriction on mortality and morbidity in a very preterm birth cohort. J Pediatr 2010; 157:733.

16. Cetin I. Amino acid interconversions in the fetal-placental unit: the animal model and human studies in vivo. Pediatr Res 2001; 49:148.

17. Bernstein IM, Horbar JD, Badger GJ, et al. Morbidity and mortality among very-low-birth-weight neonates with intrauterine growth restriction. The Vermont Oxford Network. Am J Obstet Gynecol 2000; 182:198. 
18. Lemons JA, Bauer CR, Oh W, et al. Very low birth weight outcomes of the National Institute of Child health and human development neonatal research network, January 1995 through December 1996. NICHD Neonatal Research Network. Pediatrics 2001; 107:E1.

19. Hanley GE, Janssen PA. Ethnicity-specific growth distributions for prediction of newborn morbidity. J Obstet Gynaecol Can 2012; 34:826.

20. Hanley GE, Janssen PA. Ethnicity-specific birthweight distributions improve identification of term newborns at risk for short-term morbidity. Am J Obstet Gynecol 2013; 209:428.e1.

21. Toapanta -Pinta P, Merizalde -Guerra A, Alarcón -Andrade P, Vasco-Toapanta C, Vasco- Morales S. Factores perinatales asociados a macrosomía fetal, en un hospital de Quito-Ecuador. South Florida Journal of Development 2021; 2 (4), 4945-4955

https://doi.org/10.46932/sfjdv2n4-001 